\title{
Extra-Marital Affair Can Lead Trauma and Connections in Higher Risk for Heart Disease in Indian Women
}

\author{
Rahul Hajare* \\ Indian council of Medical Research, National AIDS Research Institute, Delhi, India
}

Received: 琒July 16, 2018; Published: 制July 20, 2018

*Corresponding author: Rahul Hajare, Post Doc Scholar, Indian council of Medical Research, National AIDS Research Institute, Delhi, India

\section{Introduction}

An Extra-marital affair relationship can break heart. Extramarital affair women, according to a study, have a higher risk for heart disease. Turns out, for women, being extra-marital affair can be injurious to health. According to a study conducted by the New York University, extra-marital affair women have a higher risk for heart disease compared with non-extra-marital affair women across several modifiable risk factors [1-3]. "Our findings highlight the impact of sexual orientation, specifically sexual identity, on the cardiovascular health of women and suggest clinicians and public health practitioners should develop tailored screening and prevention to reduce heart disease risk in extra-marital affair women," Little is known about the impact of sexual orientation on heart disease risk in women, despite the fact that widow and extramarital affair women may be at a higher risk based on modifiable factors like tobacco use and poor mental health. In this study, the researchers examined differences in modifiable risk factors for heart disease and heart disease diagnoses in women of different sexual orientations. Risk factors measured included mental distress; health behaviours such as tobacco use, binge drinking, diet, and exercise; and biological risk factors such as obesity, hypertension, diabetes, and cholesterol [4]. Participants who reported having angina, coronary heart disease, heart failure, heart attack, or stroke were considered as having a diagnosis of heart disease. The researchers' analysed responses from 5000 women data ages 20 to $59[5,6]$. Differences were analysed across four groups based on their sexual identities: gay women, extra-marital affair women, heterosexual women who have sex with women, and heterosexual women. The researchers found no differences in heart disease diagnoses based on sexual orientation, but the risk for heart disease was more complicated. Gay women, heterosexual women, and heterosexual women who have sex with women had similar heart disease risk [7]. Gay women reported lower binge drinking compared with heterosexual women, but otherwise few differences in health behaviours were noted. Extra-marital affair women, however, had higher rates of several risk factors for heart disease relative to heterosexual women: mental distress, obesity, elevated blood pressure, and three different measures of diabetes (medication use, medical history, and average glycosylated haemoglobin level). "Poor mental health is a recognised risk factor for the development of heart disease," "Clinicians should be educated about sexual minority health and should routinely screen extra-marital affair women for mental distress as a risk factor for heart disease. This is particularly important as healthcare organisations increasingly include sexual orientation as part of demographic questionnaires in electronic health records [8]." The researchers also noted that the study underscores the importance of disaggregating analyses for gay and extra-marital affair participants to ascertain differences in health outcomes between these subgroups.

\section{Acknowledgement}

This study has been guided under the supervision and guidance of Renowned Immunologist Respected Dr. Ramesh S. Paranjape, Long-Time NARI Director and Renowned Scientist, India.

\section{References}

1. Rahul Hajare (2017) Can Otolaryngology Capture Window Cancer in Middle Adulthood? Ann Clin Lab Res 5(4): 205.

2. Rahul Hajare (2017) The Aggression is an Early Cause of Cancer. A Narrative Review of Classical to Modern Scientific Literature. Chronicle Med Surg 1(2): 67-68.

3. Rahul Hajare (2018) Lead Developer Angular and Effect of Force by Side Chain of Suicide Molecule in HIV AIDS Drug Discovery. Journal of Pharmaceutical Research and Reviews.

4. Rahul Hajare (2018) Safe Sex: The True Principal Health? Medical Research and Clinical Case Reports 1: 79-81.

5. Rahul Hajare (2018) Safe sex: the train your mind (revise). Gen Med Open 2(2): 1-2.

6. Rahul A Hajare (2018) 909090 Formulas and Symptoms of Adrenal Fatigue Syndrome (AFS) of Adult Men. Orthop \& Spo Med Op Acc J 1(3).

7. Rahul Hajare (2018) An Attempt to Eradicate Alcohol Dependency from Adult Men in Service Privately Managed Pharmaceutical Institutions in India. Pharmaceutical Sciences \& Analytical Research Journal 1(1): 180001.

8. Rahul H (2018) Indian Women, Trauma and Hydroxyl Drugs Dependency: Connections and Disconnections in Heart Disease for Women. Int J Curr Innov Adv Res 1(2): 1-2. 
(c) (i)

This work is licensed under Creative Commons Attribution 4.0 License

Submission Link:

Submit Article

DOI: 10.32474/DDIPIJ.2018.02.000126

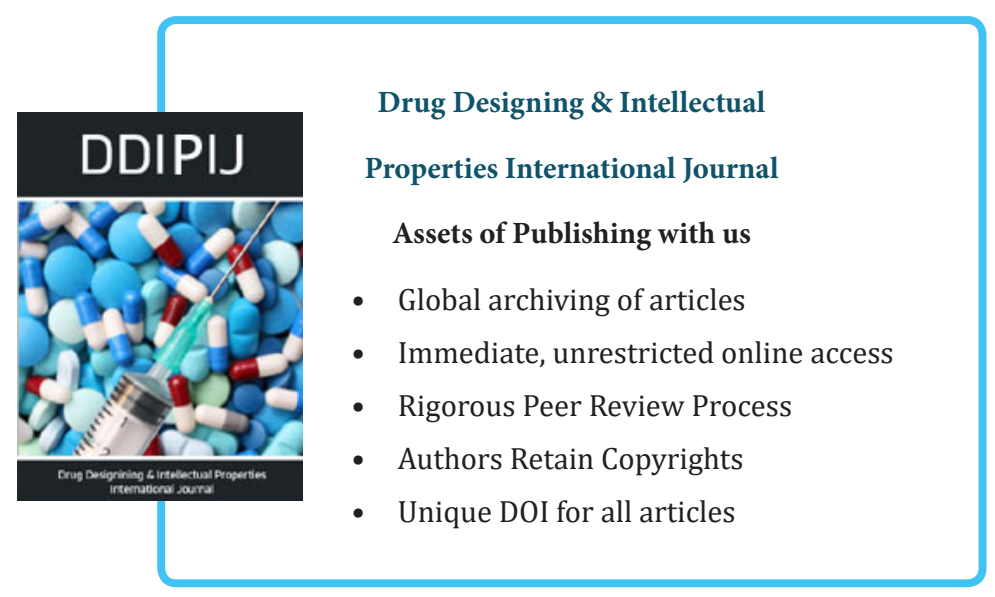

